\title{
Continuous media (CM) data stream in flash-based solid state disk (SSD) storage server
}

\begin{abstract}
Solid State Disk (SSD), a type of NAND-flash memory semiconductor device becomes popular storage systems in various types of appliances. The surveys have brought out its essential demand for replacing the classical storage devices (e.g, Magnetic disk) in a multimedia streaming server such as a Video-On-Demand (VOD) server. The most important factor in providing an efficient server is the real-time playback experience by the users. However, the NAND-flash has three issues those related to the nature of its I/O accessing procedures. This paper studies the effectiveness of proper data layout in the SSD to overcome those issues in handling the VOD server real-time playback requirement. Data allocation acts as an engine that store or retrieve the continuous media (CM) data from the server and send to users at a proper rate, good quality and offer smooth VCR functions - pause, fast/slow rewind/forward. Two allocations those utilizing the rich parallelism stripping nature in SSD, namely; (i) Sequential, and (ii) Interleaving have been examined in this study. Both allocations intelligently organize the $\mathrm{CM}$ data using the residue class algorithm and the results from the simulation experiments shown both allocations have significant outcomes in terms of the access latency and the throughput.
\end{abstract}

Keyword: VOD server; NAND-flash memory; Real-time; Data allocation; Storage 